\title{
Solitary Fibrous Tumour of Cheek: A Diagnostic Dilemma
}

\author{
Subhash Yadav* and Shubhada Kane
}

Department of Pathology, Tata Memorial Hospital, Mumbai, India

\section{ABSTRACT}

Solitary fibrous tumor (SFT), an uncommon spindle cell neoplasm is believed to be of mesenchymal origin and usually presents as pleural based mass. SFT are rare in the head-neck region but are extremely uncommon intra-orally. The patient was a young male who presented with difficulty in mouth opening. Examination revealed a submucosal nodule on the buccal surface which was histologically and immunohistochemically proven to be SFT. Authors wish to discuss the case due to its rarity and diagnostic difficulties due to many common histological mimickers at that site.

Keywords: Solitary Fibrous Tumor, Spindle Cell Neoplasm

\section{Introduction}

Solitary fibrous tumor (SFT), a spindle cell neoplasm of mesenchymal origin has particular predilection for serosal sites. It was initially stated that this was a benign variant of mesothelioma. Subsequent ultrastructural research revised this opinion and revealed that SFT originated from interstitial stem cells. ${ }^{(1)}$

Incidence of SFT in the head neck region is around $2-3 \%$. SFT within the thyroid gland, larynx, epiglottis, parapharyngeal space, major salivary glands, nasopharynx, paranasal sinuses, orbit, as well as cerebral meninges are known, but are considerably rare.$^{(1)}$ Over a period of ten years, we encountered a single case of histologically and immunohistochemistrically proven SFT of cheek. We hereby discuss a case of SFT of cheek and its diagnostic dilemma.

\section{Case History}

22-year-male, presented with localised swelling in the mouth since last 4-5 years, which was slowly and gradually increasing in size with difficulty in mouth opening. Local examination revealed a nodule measuring $1.2 \times 1 \times 1$ centimetres beneath the left buccal mucosa which was firm and slightly mobile. Overlying epithelium was unremarkable. Blood investigations were within normal limits. No radiological investigations were done. An excision biopsy was done which showed nondescript bland and uniform spindle cells dispersed among elongated, thin, parallel collagen bands in a "patternless" pattern and thick bands of collagen. On imunohistochemistry, the spindle cells were positive for CD34 and BCL2 whereas they were negative for SMA. Thus a diagnosis of SFT was rendered.

\section{Discussion}

Solitary fibrous tumor typically occurs between the fourth and eighth decade with no sex predilection. Etiopathogenesis of the tumor is still unclear. Two theories have been propsed in literature to clarify this issue. (1) Multidirectional differentiation of fibroblasts and mesenchymal multipotential cells in connective tissue. (2) Existence of specialized cells, capable of differentiation towards mesothelium. Nevertheless, most evidence advocate the theory of the mesenchymal origin of SFT. (1)

The histology of SFT has been described as "patternless pattern". Problems in diagnosis may arise due to lack of the characteristic histological features. However, three consistent microscopic features found in SFT are (a) the presence of alternate hypocellular and hypercellular areas blending into one another, (b) haphazard arrangement of ovoid to spindly cells with intervening dense collagen bands, (c) thin walled gaping and branching blood vessels. (2) The cytoplasm presents a weak eosinophilic reaction and spindly nuclei contain diffuse chromatin and small nucleoli. Numerous blood vessels presenting a typical "staghorn pattern" are common with more or less abundant infiltrates of mast cells. (1) Majority of SFTs express CD34 and Vimentin. CD99, BCL-2 and infrequently XIIIa are also positive, although this is not the rule. A negative reaction is common for $\mathrm{S}-100$ protein, cytokeratin, desmin and actin. (1)

Imunohistochemistry is extremely useful in establishing the diagnosis, no more so than CD34. It is no coincidence that since the description of CD34 expression in solitary fibrous tumors, there has been a flurry of case reports in a wide range of sites. Cytogenetic and fluorescence in 


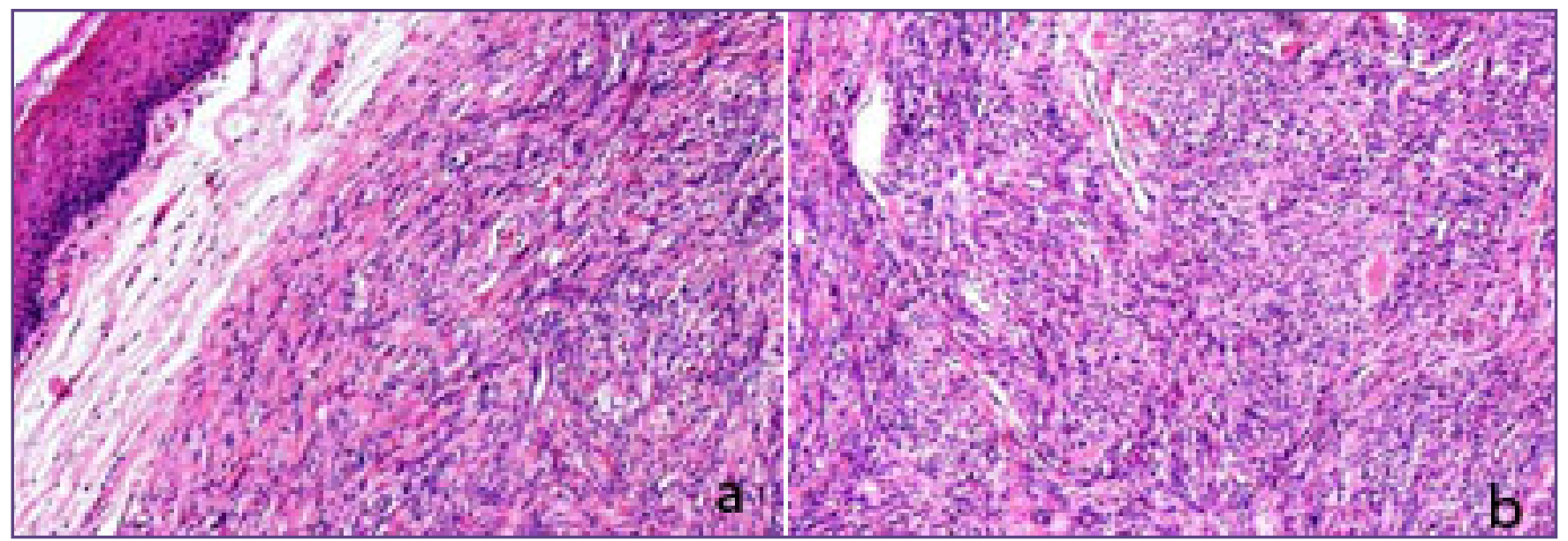

Fig. 1: a. Cellular tumor with a vague intersecting pattern located beneath the epithelium. The overlying epithelium is unremarkable. (HE, $x$ 40) b. Cellular tumor showing thin walled capillaries amongst the tumor cells. (HE, $x$ 100).

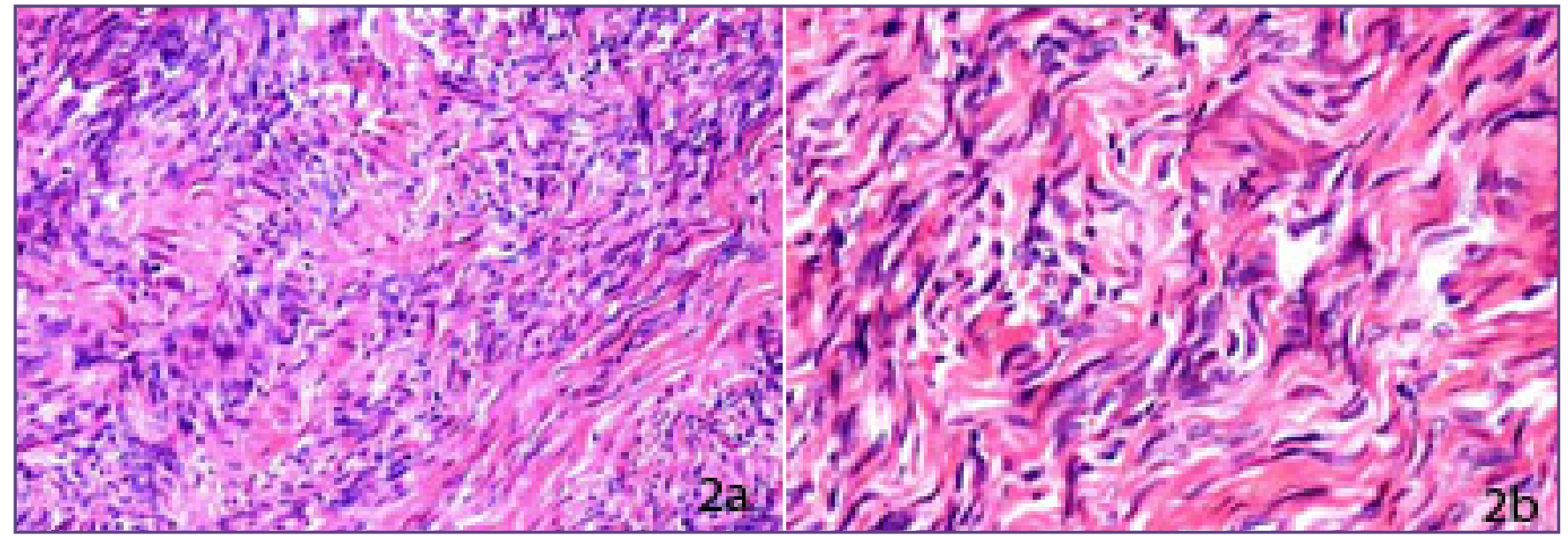

Fig. 2: a. Tumor is composed of spindle cells without much cytological atypia. Necrosis, hemorrhage and significant mitosis are not seen. (HE, $x$ 400) b. Thick ropy collagen interspersed with the tumor cells noted. (HE, x 400).

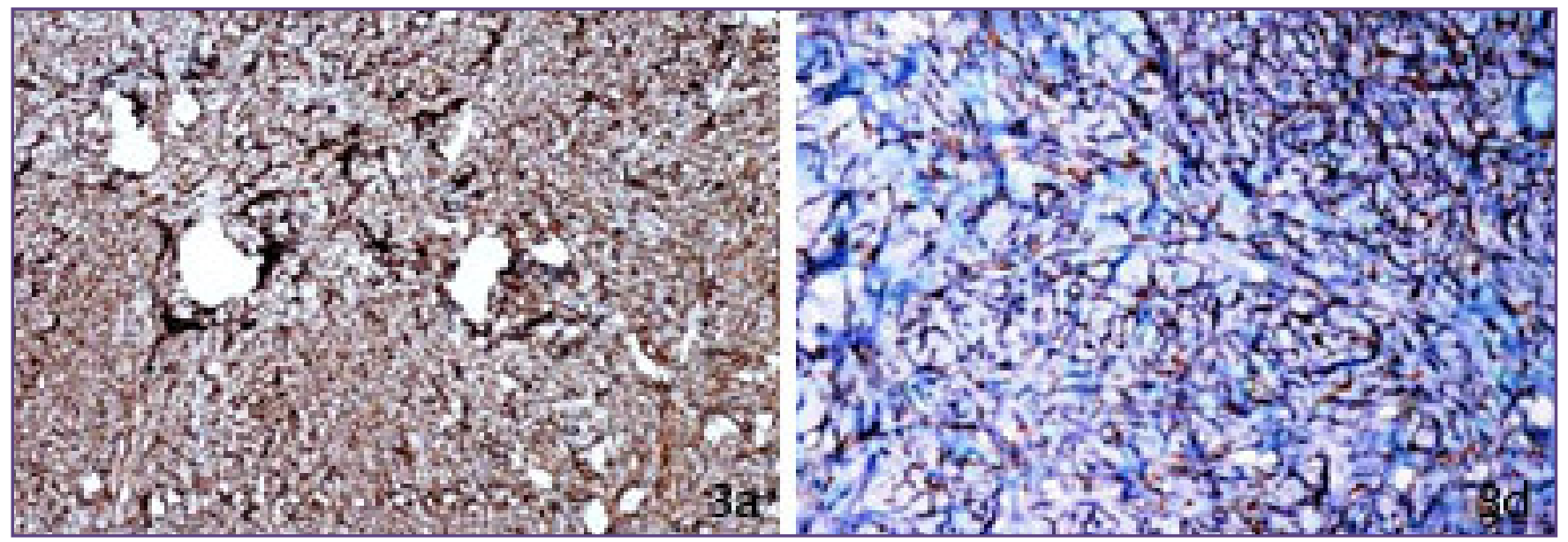

Fig. 3: a. Tumour cells strongly \&diffusely express Vimentin. (IHC, $x$ 400x) b. BCL-2 is positive in the tumour cells. (IHC, $x$ 400x). 


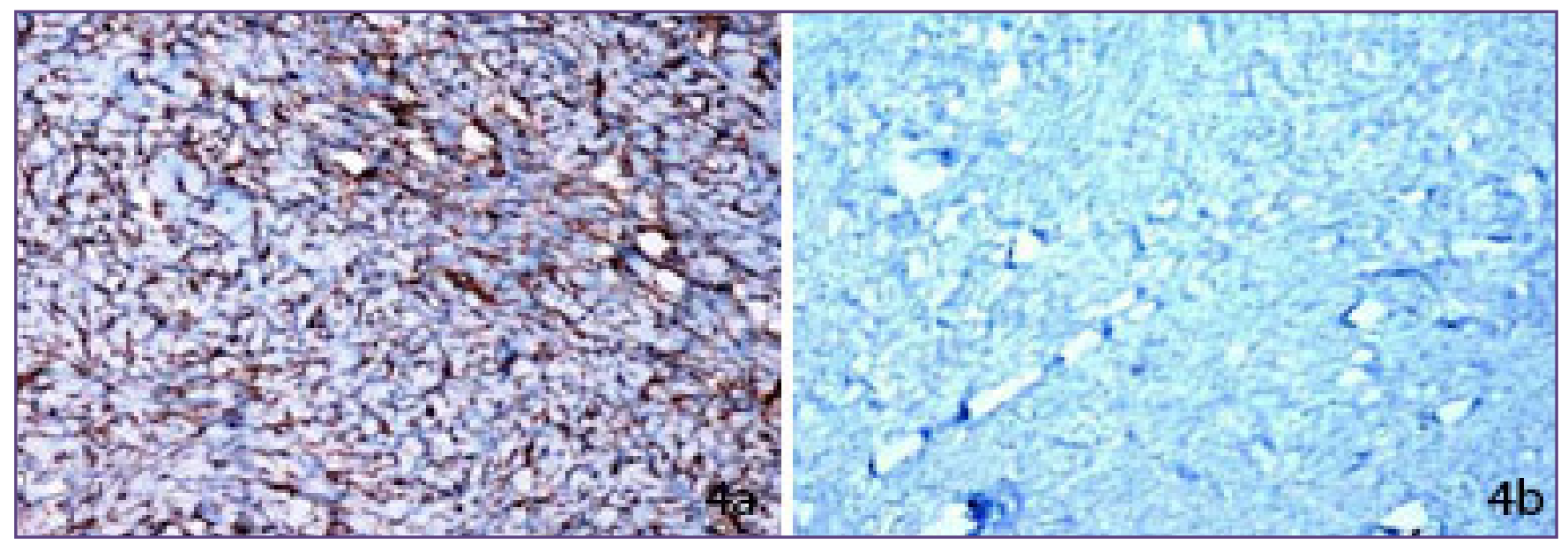

Fig. 4: a. CD34 shows strong diffuse positivity. (IHC, x 400x) b. S100 protein is negative. (IHC, $\mathrm{x} 400 \mathrm{x}$ ).

situ hybridization has shown no specific chromosomal abnormality and, unlike a growing number of sarcomas, molecular tests are not utilised in confirming the diagnosis. (3)

Differential diagnosis of SFT relates to other mesenchymal tumors, including hemangiopericytoma, myofibroblastoma, schwannoma, neurofibroma, leiomyoma, as well as inflammatory disorders such as nodular fasciitis. The most challenging issue is the differentiation between SFT and hemangiopericytoma due to very similar histological and immunohistochemical profiles. It is believed that hemangiopericytoma is more homogenous. It also contains fewer collagen fibers and more cells compared to SFT. Positive CD34 staining tends to be more focal and less regular in cases of hemangiopericytoma. (4)

Spindle cell neoplasms of neuronal origin, including schwannoma and neurofibroma, display some clinical and immunohistochemical attributes similar to SFT. These are fairly well circumscribed from surrounding tissues due to capsule around the tumor. [10]. Distinct positive staining for S-100 protein, which is typical of nerve sheaths, is a general immunohistochemical hallmark of neuronal tumors. As mentioned above, SFT displays a negative reaction for S-100 protein, which disqualifies its neuronal origin. $(5,6)$

Leiomyoma is characterized by more fascicular and regular arrangements of spindly cells compared to SFT. Ultrastructural and immunohistochemistry helps in proper diagnosis of leiomyoma so it should not be a challenge.(5)

Fibromatosis is a benign fibroblastic lesion with increased cellularity and infiltrating margins that lack alternate hypocellular and hypercellular areas and short spindly cells seen in SFT. The vessels in fibromatosis are venule sized, thick walled interspersed throughout the lesion in contrast to SFT that has branching vessels. It lacks CD34 positivity. (2).

It has been proven that chromosomal aberrations typical of SFT may be characterized by additional chromosomes $2,7,8,9,10,18$ and 21 in the tumor cells, additional arms of chromosomes 12p, 12q and 15q, loss of arms 4q, 5p, 6 , $9 p, 13 q, 15 q$, and 20p, loss of entire chromosomes 6,10 , $17,21,22$ and $X$, as well as complex translocations within chromosomes 1, 17 and 18. It has been also stated that the most frequent chromosomal aberration in SFT is the loss of arm 13q. The significance of this abnormality and its influence on the development and biology of the tumor has not been established yet. $(8,9)$

\section{Conclusion}

SFT is a recently described rare soft tissue neoplasm occurring at pleural and extra-pleural sites. However intraoral location is very rarely described. Because of many close mimickers of SFT, a diagnosis should not be made only on histology but with histology substantiated with immunohistochemistry.

\section{References}

1. Chomik P, Michcik A, Michajłowski I, Sobjanek M, Włodarkiewicz A Solitary fibrous tumor in the oral cavity: a case report and diagnostic dilemma Postep Derm Alergol 2012; XXIX, 5: 395-400

2. Ghosh S, Shet TM, Chinoy RF, Kane SV. Solitary fibrous tumour: a diagnostic dilemma. Indian J Pathol Microbiol. 2007 Jul;50(3):520-4. PubMed PMID: 17883122.

3. Langman G. Solitary fibrous tumor: A pathological enigma and clinical dilemma. J Thorac Dis. 2011 June; 3(2): 86-87.

4. Shine N, nor nurul Khasri M, Fitzgibbon J, O'Leary G. Solitary fibrous tumor of the floor of the mouth: case 
report and review of the literature. Ear Nose Throat J. 2006 Jul;85(7):437-9. Review. PubMed PMID: 16909814.

5. Wu SL, Vang R, Clubb FJ Jr, Connelly JH. Solitary fibrous tumor of the tongue:report of a case with immunohistochemical and ultrastructural studies. Ann Diagn Pathol. 2002 Jun;6(3):168-71. PubMed PMID: 12089728.

6. Hirano M, Tanuma J, Shimoda T, Sugihara K, Tsuneyoshi M, Kitano M. Solitary fibrous tumor in the mental region. Pathol Int. 2001 Nov;51(11):905-8. PubMed PMID: 11844061.
7. Yadav AK, Nadarajah J, Chandrashekhara SH, Tambade VD, Acharya S. Myoepithelioma of the soft palate: a case report. Case Rep Otolaryngol.2013;2013:642806.

8. Manor E, Bodner L. Chromosomal aberrations in oral solitary fibrous tumor. Cancer Genet Cytogenet. 2007 Apr 15;174(2):170-2. PubMed PMID: 17452261.

9. Martin AJ, Summersgill BM, Fisher C, Shipley JM, Dean AF. Chromosomal imbalances in meningeal solitary fibrous tumors. Cancer Genet Cytogenet. 2002 Jun;135(2):160-4. Review. PubMed PMID: 12127400.

*Corresponding author:

Dr. Subhash Yadav, Department of Pathology, Tata Memorial Hospital, Mumbai, India

Phone: +91 022-24177260

Email: drsubhashyadav23@gmail.com

Date of Submission : 09/01/2019

Date of Acceptance : 11/05/2020

Financial or other Competing Interests: None.
Date of Publication : 30/06/2020 